\title{
Interface sharpening in miscible and isotopic multilayers: Role of short-circuit diffusion
}

\author{
A. Tiwari, ${ }^{1}$ M. K. Tiwari, ${ }^{2}$ M. Gupta, ${ }^{3}$ H.-C. Wille,${ }^{4}$ and A. Gupta ${ }^{1, *}$ \\ ${ }^{1}$ Amity Centre for Spintronic Materials, Amity University, Uttar Pradesh, Sector 125, Noida 201313, India \\ ${ }^{2}$ Raja Ramanna Centre for Advanced Technology, Indore 452013, India \\ ${ }^{3}$ UGC-DAE, Consortium for Scientific Research, Indore 452017, India \\ ${ }^{4}$ Deutsches Elektronen-Synchrotron DESY, Notkestraße 85, 22607 Hamburg, Germany
}

(Received 17 October 2018; revised manuscript received 6 March 2019; published 10 May 2019)

\begin{abstract}
Atomic diffusion at nanometer length scale may differ significantly from bulk diffusion, and may sometimes even exhibit counterintuitive behavior. In the present work, taking $\mathrm{Cu} / \mathrm{Ni}$ as a model system, a general phenomenon is reported which results in sharpening of interfaces upon thermal annealing, even in miscible systems. Anomalous $\mathrm{x}$-ray reflectivity from a $\mathrm{Cu} / \mathrm{Ni}$ multilayer has been used to study the evolution of interfaces with thermal annealing. Annealing at $423 \mathrm{~K}$ results in sharpening of interfaces by about $38 \%$. This is the temperature at which no asymmetry exists in the interdiffusivities of $\mathrm{Ni}$ and $\mathrm{Cu}$. Thus, the effect is very general in nature, and is different from the one reported in the literature, which requires a large asymmetry in the diffusivities of the two constituents [Erdélyi et al., Science 306, 1913 (2004)]. The general nature of the effect is conclusively demonstrated using isotopic multilayers of ${ }^{57} \mathrm{Fe} /{ }^{\text {natural }} \mathrm{Fe}$, in which evolution of isotopic interfaces has been observed using nuclear resonance reflectivity. It is found that annealing at suitably low temperature (e.g., $523 \mathrm{~K}$ ) results in the sharpening of the isotopic interfaces. Since chemically it is a single Fe layer, any effect associated with concentration dependent diffusivity can be ruled out. The results can be understood in terms of fast diffusion along short-circuit paths like triple junctions, which results in an effective sharpening of the interfaces at relatively low temperatures.
\end{abstract}

DOI: 10.1103/PhysRevB.99.205413

\section{INTRODUCTION}

Interfaces hold the key to functionality of multilayer nanostructures. Various phenomena like tunnel magnetoresistance, exchange bias, spin-orbit torque, and anisotropic Dzyalosinskii-Moriya interactions depend crucially on the structure of the interfaces [1-3]. Therefore, a controlled manipulation of the interface structure is a prime requirement for tuning the functionality of multilayer nanostructures. Atomically sharp interfaces are an essential requirement for several applications, for example (i) in the case of x-ray and neutron multilayer mirrors, sharp interfaces are a prerequisite in order to have high reflectivity at the Bragg peak [4,5], (ii) in a magnetic tunnel junction, magnetoresistance sensitively depends upon the sharpness of interfaces between magnetic electrodes and tunnel barrier [6], (iii) sharp interfaces are also a prerequisite for interface induced perpendicular magnetic anisotropy in systems like $\mathrm{CoFeB} / \mathrm{MgO}, \mathrm{Co} / \mathrm{Pt}$ [7-10]. This necessitates a detailed understanding of the interdiffusion at the interfaces occurring during film deposition as well as during postdeposition treatments like thermal annealing.

Typical length scales involved in such devices are in the sub-nm range. Extensive studies in the literature show that interdiffusion at this length scale can be very different from that in the bulk [11-16]. Even the well-established laws of diffusion may not hold at $\mathrm{nm}$ length scale, as these laws are derived assuming a continuum medium, while at $\mathrm{nm}$

*agupta2@amity.edu length scale, the discrete nature of atomic lattice becomes important [11]. Several counter-intuitive behaviors have been observed at nm length scale: (i) As a rule, grain-boundary (GB) diffusion is faster than volume diffusion due to the higher density of defects in the GB region. However, volume diffusion in the nanocrystalline $\mathrm{Fe}_{73.5} \mathrm{Si}_{13.5} \mathrm{~B}_{9} \mathrm{Nb}_{3} \mathrm{Cu}_{1}$ alloy is found to be faster than GB diffusion [12]. (ii) In the nanocrystalline FeN phase, self-diffusion of Fe atoms, which are much bigger in size, is faster than $\mathrm{N}$ diffusion [13]. (iii) Strong asymmetry of interdiffusion has been observed at the two interfaces of a binary system like Fe-Si. It is found that diffusion at the $\mathrm{Fe}$-on-Si interface is faster than at the $\mathrm{Si}$-on- $\mathrm{Fe}$ interface [14]. (iv) In completely miscible systems like $\mathrm{Ni} / \mathrm{Cu}$ or Mo/ $\mathrm{V}$, with a large asymmetry in diffusivities of the two constituents, a sharpening of interfaces has been observed instead of intermixing $[15,16]$.

Atomic diffusion in the $\mathrm{Ni} / \mathrm{Cu}$ system has been extensively studied in the literature [10,11,14-19]. A large asymmetry exists between volume diffusivities of $\mathrm{Cu}-\mathrm{in}-\mathrm{Ni}$ and $\mathrm{Ni}-\mathrm{in}-\mathrm{Cu}$. This asymmetry results in composition dependent diffusivities which in turn cause interfaces to sharpen on thermal annealing $[15,20]$. X-ray-diffraction studies on coherent $\mathrm{Cu} / \mathrm{Ni}$ multilayers have evidenced layer by layer diffusion of $\mathrm{Ni}$ at the interfaces [18]. All these studies have been done at sufficiently high temperatures at which volume diffusivity is appreciable. The associated diffusion length in this region is in the range of a few $\mathrm{nm}$. On the other hand for most of the applications of such multilayers, e.g., in spintronic devices, x-ray and neutron mirrors, etc., typical layer thicknesses themselves are in the range of a few $\mathrm{nm}$ and even sub-nm diffusion lengths 


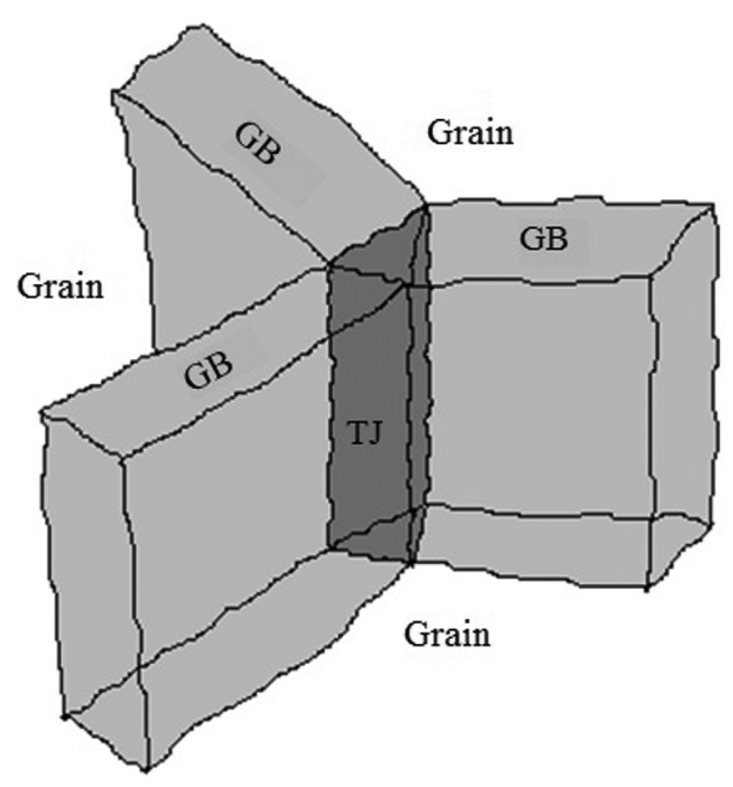

FIG. 1. Schematic representation of grains, grain boundaries, and triple junction.

can significantly alter the interface structure and hence the functional properties of multilayers. Thus, it is important to understand the atomic level processes involved in interdiffusion at relatively low temperatures which are encountered during processing and/or operation of such multilayer devices.

In the present work, we report a more general phenomenon in multilayers, which results in sharpening of interfaces even in miscible systems when there is no asymmetry in diffusivities of the two constituents. Taking $\mathrm{Cu} / \mathrm{Ni}$ as a model system, it is shown that interface sharpening can occur even at temperatures well below the temperatures at which volume diffusion becomes appreciable, and the average diffusion lengths are in the sub-nm range. This is the temperature range in which one does not expect any asymmetry between diffusivities of $\mathrm{Cu}$ in- $\mathrm{Ni}$ and $\mathrm{Ni}-\mathrm{in}-\mathrm{Cu}$, and therefore the mechanism proposed in the literature for the interface sharpening should not be operative. We propose that the atomic level processes involved in the observed effect are very different, and highlight the role of short-circuit diffusion along triple junctions in the temperature range of interest for processing of multilayer nanostructure devices. A triple junction is a line defect where three grains and grain boundaries meet (Fig. 1) [21]. In a polycrystalline material, the intergranular region consists primarily of grain boundaries, which form a three-dimensional (3D) network, isolating individual crystallites from one another. Similarly, facets of grain boundaries are connected to one another at triple junction lines, which form a fully interconnected matrix without any dead ends. Mechanisms of atomic diffusion within the crystallites (volume diffusion), in the grainboundary region and along the triple junction lines are very different from one another [22-24]. With highly disordered structure, triple junctions have diffusivities that are several orders of magnitude higher [25,26] than grain-boundary diffusivity, which in turn is generally significantly higher than volume diffusivity. In general, in polycrystalline materials, the volume fraction occupied by triple junctions is small and their contribution to atomic diffusion can be neglected. However, as the crystallite size decreases to nanoscale, the volume fraction occupied by them and hence their contribution to atomic diffusion becomes appreciable; anomalously high atomic diffusion in nanocrystalline materials, even beyond what is expected from a high density of equilibrated grain boundaries, could be explained in terms of short-circuit diffusion along triple junctions [26].

More convincing evidence for the proposed mechanism of interface sharpening in the present case is obtained from the observed sharpening of the isotopic interfaces in a ${ }^{57} \mathrm{Fe} /{ }^{\text {natural }} \mathrm{Fe}$ multilayer as observed using nuclear resonance reflectivity. Since in this case both types of the layers are chemically identical, there is no question of any concentration dependent diffusivity.

These results are also of practical importance since in general, x-ray and neutron mirrors, and magnetic multilayer for spintronic applications have typical layer thicknesses in the $\mathrm{nm}$ range, and their properties can get modified drastically even if the interfaces are modified at the sub-nm length scale.

\section{EXPERIMENTAL DETAILS}

The multilayer of $\mathrm{Cu} / \mathrm{Ni}$ system with nominal structure: substrate $/[\mathrm{Cu}(3.28 \mathrm{~nm}) / \mathrm{Ni}(2.2 \mathrm{~nm})]_{10}$ and the isotopic multilayer of ${ }^{57} \mathrm{Fe} /{ }^{\text {natural }} \mathrm{Fe}$ having nominal structure: substrate $/\left[{ }^{57} \mathrm{Fe}(3.2 \mathrm{~nm}) /{ }^{\text {natural }} \mathrm{Fe}(3.2 \mathrm{~nm})\right]_{10}$ were prepared by ion beam sputtering at room temperature on $\mathrm{Si}$ (111) substrates. The substrate had a surface roughness of $0.56 \mathrm{~nm}$ and a 1.5-nm-thick oxide layer at the surface, as determined using $\mathrm{x}$-ray reflectivity. Base pressure in the chamber was $2 \times 10^{-8}$ mbar. The chamber was flushed with $5 \mathrm{~N}$ purity $\mathrm{Ar}$ gas several times before starting the deposition. The base partial pressure of oxygen in the chamber as determined using a MKS residual gas analyzer (model EVE-110-001) was $1 \times 10^{-9} \mathrm{mbar}$. For the deposition of multilayer structure, the targets of $\mathrm{Cu}$ (99.99\% purity) and $\mathrm{Ni}(99.99 \%$ purity) were alternatively sputtered by an Ar ion beam of $1000 \mathrm{eV}$ from a $3-\mathrm{cm}$ broad beam Kaufman-type ion source. For preparation of isotopic multilayers targets of ${ }^{\text {natural }} \mathrm{Fe}$ (99.99\% purity) and ${ }^{57} \mathrm{Fe}(99.95 \%$ purity and $95 \%$ enrichment) were used. In the preparation of both the multilayer structures, the flow of $\mathrm{Ar}$ gas in the deposition chamber was controlled by a mass flow controller (MKC-MFC 1179A) at 5 SCCM (standard cubic centimeters per minute). Oxygen partial pressure in the chamber during deposition remained in the range of $10^{-9}$ mbar.

In order to achieve variation in grain size in the isotopic multilayer, another isotopic multilayer having structure substrate $/{ }^{\text {natural }} \mathrm{Fe}(50 \mathrm{~nm}) /\left[{ }^{57} \mathrm{Fe}(1.8 \mathrm{~nm}) /{ }^{\text {natural }} \mathrm{Fe}(3.8 \mathrm{~nm})\right]_{10}$ was prepared at an elevated substrate temperature of $573 \mathrm{~K}$, using direct current magnetron sputtering at a constant power of $50 \mathrm{~W}$. The base pressure in the chamber was $1.7 \times$ $10^{-7} \mathrm{mbar}$, while the pressure during deposition was $3.4 \times$ $10^{-3}$ mbar. The flow of Ar gas in the deposition chamber was kept at 50 SCCM.

$\mathrm{X}$-ray-diffraction measurements were done using Bruker D5000 diffractometer in $\theta-2 \theta$ geometry. Anomalous x-ray reflectivity measurements have been done at beamline BL16 of Indus- 2 in order to study the interdiffusion in $\mathrm{Cu} / \mathrm{Ni}$ 
multilayers. This technique is capable of measuring diffusion length down to an accuracy of $0.1 \mathrm{~nm}$. Since the electron density contrast between $\mathrm{Cu}$ and $\mathrm{Ni}$ is negligible, $\mathrm{x}$-ray reflectivity measurements have been done across the $\mathrm{K}$ absorption edges of $\mathrm{Ni}$, so as to increase the x-ray-scattering contrast. It may be noted that the refractive index of Ni would vary rapidly with energy around the absorption edge therefore even a small deviation in the $\mathrm{x}$-ray energy from the designated value can cause significant variation in the refractive index. In order to avoid a possible artifact in the fitting of the reflectivity pattern due to some deviation in the $\mathrm{x}$-ray energy from the designated value, measurements were done at five different x-ray energies across the absorption edge of $\mathrm{Ni}$, namely, 8290, $8315,8340,8365$, and $8390 \mathrm{eV}$. Simultaneous fitting of all five reflectivity patterns using the same structural parameters for the multilayer provides much more reliable and unambiguous fitting of the data. The refractive indices of $\mathrm{Cu}$ and $\mathrm{Ni}$ at various energies were taken from CXRO data tables [27]. The fitting of reflectivity data was done using Parratt's formalism [28].

Variation in the roughness of isotopic interfaces in ${ }^{57} \mathrm{Fe} /{ }^{\text {natural }} \mathrm{Fe}$ multilayer was studied using nuclear resonance reflectivity (NRR) measurements done at the Dynamics Beamline P01 at PETRA-III, DESY, Hamburg. To perform NRR measurements, the energy of the radiation was kept at 14.4 KeV (Mössbauer resonance of ${ }^{57} \mathrm{Fe}$ ) in 40 bunch mode with the bunch separation of 192 ns. The delayed events, resulting from the nuclear forward scattering, were counted by a fast avalanche photodiode (APD) detector.

The isotopic multilayer sample was cut into $10 \times 10 \mathrm{~mm}$ pieces and each piece was annealed at a particular temperature for $1 \mathrm{~h}$. Cu/Ni multilayer samples were isochronally annealed at different temperatures in a tubular furnace having a uniform temperature zone of $5 \mathrm{~cm}$. The pressure in the tube was $1 \times$ $10^{-6} \mathrm{mbar}$ and the temperature was maintained within $\pm 1^{\circ} \mathrm{C}$ of the set temperature during annealing.

\section{RESULTS AND DISCUSSIONS}

\section{A. $\mathrm{Cu} / \mathrm{Ni}$ multilayer}

Figure 2 gives the $\mathrm{x}$-ray-diffraction pattern of as-prepared $\mathrm{Cu} / \mathrm{Ni}$ multilayer taken in $\theta-2 \theta$ geometry, with the scattering vector normal to the film surface. One observes a dominant peak around $2 \theta=44^{\circ}$. Both $\mathrm{Ni}$ as well as $\mathrm{Cu}$ have fcc structure with their (111) peak at $45^{\circ}$ and $43^{\circ}$ respectively. The observed peak is in between these angular positions. The crystallite size as obtained from the width of diffraction peak using Scherrer formula comes out to be $9.7 \mathrm{~nm}$, which is about four times the thickness of an individual $\mathrm{Ni}$ or $\mathrm{Cu}$ layer. This means that a crystallite, on the average, extends over four adjacent layers, suggesting a partial coherency between layers of $\mathrm{Ni}$ and $\mathrm{Cu}$. Appearance of weak sidebands around the (111) peak with separation from the main peak corresponding to the periodicity of the multilayer is also suggestive of partial coherence between $\mathrm{Ni}$ and $\mathrm{Cu}$ grains. At such a small grain size of $9.7 \mathrm{~nm}$, even the grain boundaries and triple junction defects would occupy a significant volume fraction of the film. Taking the typical width of the grain boundaries to be $1 \mathrm{~nm}$, the volume fraction occupied by grain boundaries and triple

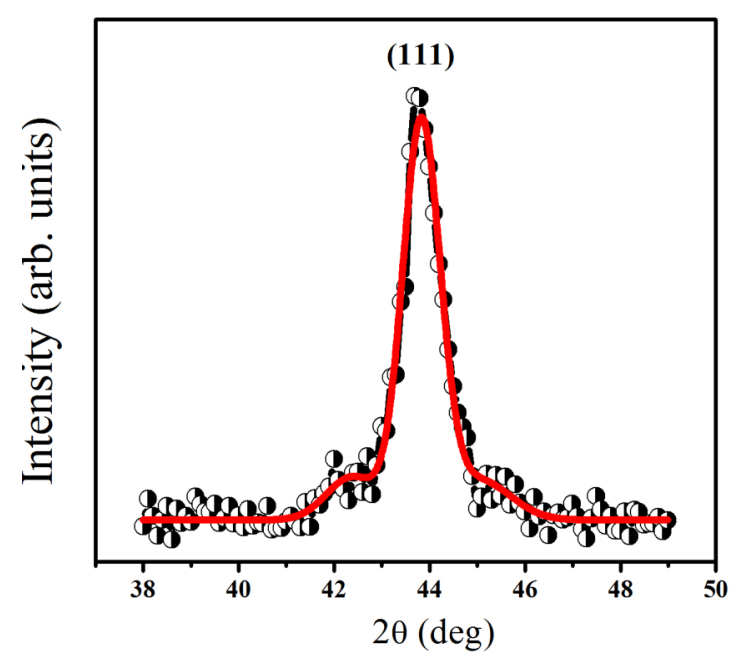

FIG. 2. $\mathrm{XRD}$ of $\mathrm{Cu} / \mathrm{Ni}$ multilayer in the as-prepared state.

junctions can be calculated to be $25 \%$ and $3 \%$ respectively [29].

Figure 3(a) gives the $\mathrm{x}$-ray reflectivity of pristine $\mathrm{Cu} / \mathrm{Ni}$ multilayer at five different energies across the absorption edge of Ni. Energies of 8290 and $8315 \mathrm{eV}$ are below the absorption edge of $\mathrm{Ni}$ and $8340,8365,8390 \mathrm{eV}$ are above the absorption edge of Ni. A variation in the refractive indices and hence in the scattering contrast between $\mathrm{Ni}$ and $\mathrm{Cu}$ occurs as the energy of the $\mathrm{x}$ rays varies, the contrast being a maximum at $8340 \mathrm{eV}$, which is just above the absorption edge of $\mathrm{Ni}$. The height of the Bragg peak exhibits a systematic variation with energy because of the variation in the scattering contrast. All five reflectivity patterns were fitted simultaneously by taking the same set of values of parameters like thicknesses and electron densities of $\mathrm{Ni}$ and $\mathrm{Cu}$ layers and their interface roughnesses.

Figure 3 (b) gives the $\mathrm{x}$-ray reflectivity pattern of $\mathrm{Cu} / \mathrm{Ni}$ multilayer isochronally annealed at various temperatures for 1 $\mathrm{h}$ each. Results are presented for an x-ray energy of $8340 \mathrm{eV}$. The continuous curves in the figure represent the best fit to the experimental data. The results of fitting are summarized in Table I. One can see that the roughness of $\mathrm{Cu}$-on-Ni is significantly higher than that of Ni-on-Cu. This result is in general agreement with the literature [16]. With thermal annealing, the only parameter which exhibits significant variation is the interface roughness. One expects that with thermal annealing as the interdiffusion between $\mathrm{Cu}$ and $\mathrm{Ni}$ layers occurs, the interface roughnesses would increase and the height of the Bragg peak should come down. One can calculate the diffusion length from the relative decrease in the height of the first Bragg peak using the relation [30,31]

$$
\ln \left[\frac{I(t)}{I(0)}\right]=\frac{-8 \pi^{2} D(T) t}{d^{2}}
$$

where $I(0)$ and $I(t)$ are the intensities of the Bragg peak before and after annealing at temperature $T$ for a time $t . D(T)$ is the diffusivity at temperature $T$ and $d$ is the bilayer period.

Surprisingly after annealing at $423 \mathrm{~K}$ for $1 \mathrm{~h}$, the height of the Bragg peak exhibits an enhancement relative to that in the as-deposited multilayer, suggesting suppression in the interface roughnesses. Beyond $423 \mathrm{~K}$ the Bragg peak exhibits 

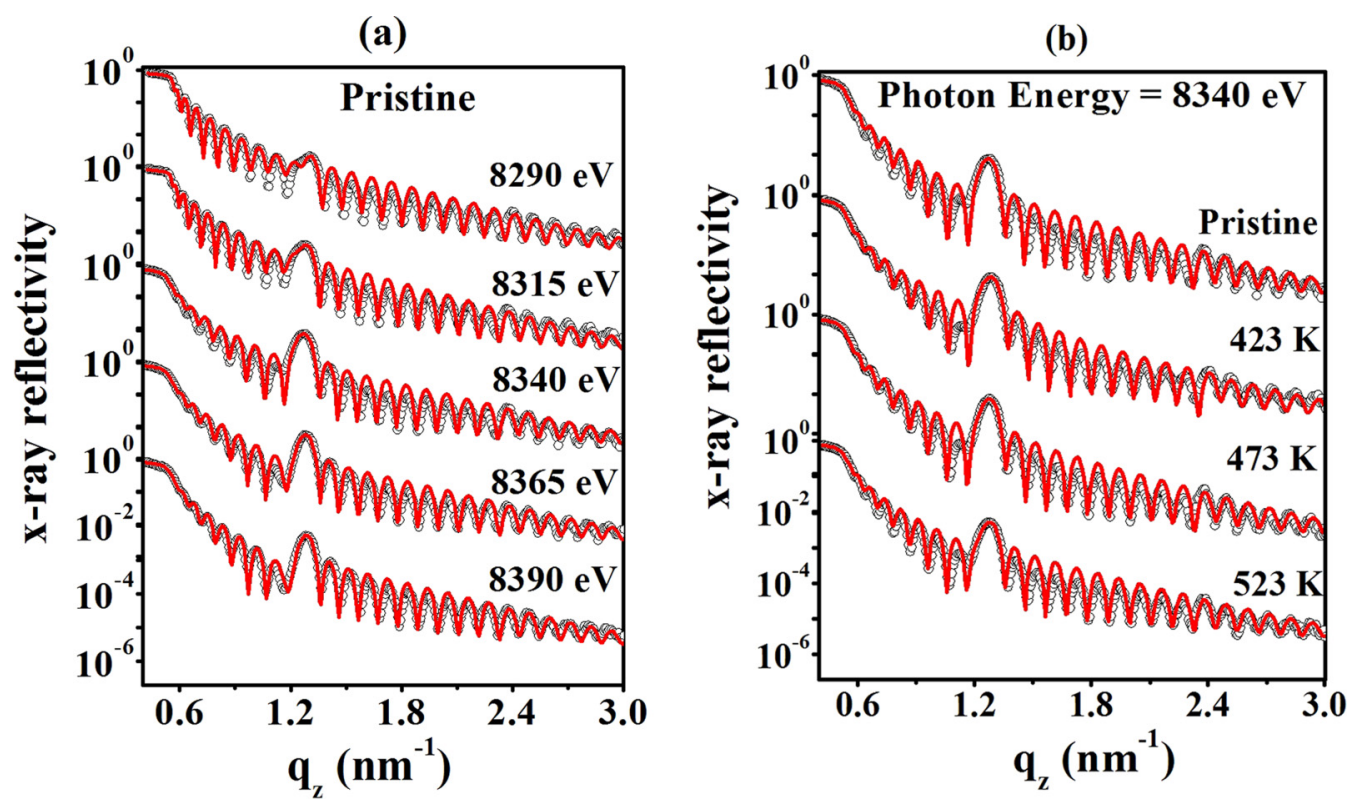

FIG. 3. (a) X-ray reflectivity of pristine $\mathrm{Cu} / \mathrm{Ni}$ multilayer taken at different $\mathrm{x}$-ray energies across the absorption edge of Ni. The continuous curves represent the best fit to the data. (b) X-ray reflectivity of $\mathrm{Cu} / \mathrm{Ni}$ multilayer as a function of isochronal annealing at different temperatures for $1 \mathrm{~h}$ each. The continuous curves represent the best fit to the data. Various reflectivity curves are shifted vertically with respect to each other for the clarity of presentation.

a systematic reduction in the height, as expected on the basis of interdiffusion. Figure 4 gives the diffusivity at 473 and $523 \mathrm{~K}$ as calculated using Eq. (1). For comparison the volume, grain-boundary, and triple junction diffusivities in the $\mathrm{Cu} / \mathrm{Ni}$ system taken from literature [32,33] are also shown. One can see that calculated diffusivities lie roughly along the line for grain-boundary diffusion.

On another piece of the multilayer specimen, reflectivity measurements were done as a function of the annealing time at $423 \mathrm{~K}$. Figure 5 gives the reflectivity pattern of the specimen after annealing at $423 \mathrm{~K}$ for $30 \mathrm{~min}$, at which the maximum increase in the height of the Bragg peak was observed. It may be noted that the piece of the sample used for isothermal annealing had a bilayer period $\sim 4 \%$ smaller than that of the piece used for isochronal annealing, causing a small relative shift of the position of the Bragg peak in the two cases. The inset in Fig. 5 compares the height of the first-order Bragg peak after different annealing temperatures on a linear scale. The reflectivity at the first-order Bragg peak increases from $0.7 \%$ in as-prepared multilayer to $2 \%$ after 30 min annealing, and corresponding decrease in the interface roughness is from 1.27 to $0.65 \mathrm{~nm}$ on the Ni-on-Cu interface and from 1.6 to $1.1 \mathrm{~nm}$ on the $\mathrm{Cu}-\mathrm{on}-\mathrm{Ni}$ interface respectively. Annealing for longer time results in a decrease in the reflectivity. Diffusivity at $423 \mathrm{~K}$ was calculated by taking the decrease in the height of the Bragg peak after $60 \mathrm{~min}$ annealing relative to that after $30 \mathrm{~min}$ and is also shown in Fig. 4. One may note that the calculated diffusivity comes out to be orders of magnitude higher than the extrapolated value of grain-boundary diffusion at this temperature.

It may be noted that both experimental as well as theoretical studies have shown that in miscible systems having a large asymmetry in the diffusivities of the two constituents, diffusion can result in sharpening of the interfaces. In the $\mathrm{Mo} / \mathrm{V}$ system, annealing up to $973 \mathrm{~K}$ resulted in sharpening of interfaces from 1.7 and $1.4 \mathrm{~nm}$ respectively to $0.78 \mathrm{~nm}$ [15]. In the $\mathrm{Ni} / \mathrm{Cu}$ system also, interface sharpening has been observed upon annealing at $773 \mathrm{~K}$ [16]. The observed effect has been attributed to large asymmetry in the diffusivities of the two constituents, which leads to a concentration dependent

TABLE I. Results from the fitting of anomalous $\mathrm{x}$-ray reflectivity of $\mathrm{Cu} / \mathrm{Ni}$ multilayer as a function of isochronal annealing at different temperatures for $1 \mathrm{~h}$ each. The results of the sample annealing at $423 \mathrm{~K}$ at $0.5 \mathrm{~h}$ are also included.

\begin{tabular}{|c|c|c|c|c|c|c|c|}
\hline $\begin{array}{l}\text { Temperature } \\
\text { (K) }\end{array}$ & \multicolumn{3}{|c|}{$\mathrm{Cu}$} & \multicolumn{3}{|c|}{$\mathrm{Ni}$} & $\operatorname{chi}^{2}$ \\
\hline RT & $3.27 \pm 0.05$ & $1.27 \pm 0.05$ & $6.38 \times 10^{-4}$ & $2.2 \pm 0.05$ & $1.6 \pm 0.05$ & $4.4 \times 10^{-4}$ & 1.215 \\
\hline $423(0.5 \mathrm{~h})$ & $3.2 \pm 0.05$ & $0.65 \pm 0.05$ & $6.28 \times 10^{-4}$ & $2.1 \pm 0.05$ & $1.1 \pm 0.05$ & $4.5 \times 10^{-4}$ & 0.216 \\
\hline 423 & $3.24 \pm 0.05$ & $1.08 \pm 0.05$ & $6.28 \times 10^{-4}$ & $2.18 \pm 0.05$ & $1.45 \pm 0.05$ & $4.5 \times 10^{-4}$ & 0.726 \\
\hline
\end{tabular}




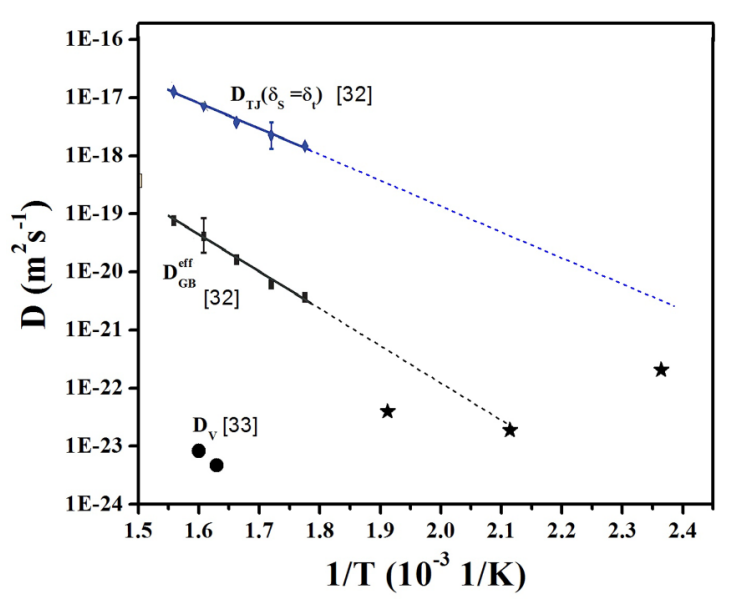

FIG. 4. Coefficients for interdiffusion in $\mathrm{Cu} / \mathrm{Ni}$ system for volume, grain-boundary, and triple junction diffusion taken from the literature [32,33]. The dashed lines represent extrapolation of literature data to lower temperatures. Stars represent the average diffusivities at different temperatures as obtained in the present work. Typical error bars in the calculated values are about $10 \%$.

diffusivity in these systems. With increasing $\mathrm{Ni}$ concentration the diffusivity of $\mathrm{Cu}$ decreases while that of $\mathrm{Ni}$ increases. Also, studies have shown that while the volume diffusivities of $\mathrm{Cu}$ and $\mathrm{Ni}$ are very different, no such asymmetry exists in grain-boundary or triple junction diffusivities of the two constituents [19]. Thus the observed interface sharpening in the literature is a consequence of a large asymmetry in the volume diffusivities of the two constituents. Indeed the temperature regime in which interface sharpening has been observed in the literature is such that volume diffusion is appreciable. In contrast, in the temperature range studied in the present work, volume diffusivity is negligible. At $423 \mathrm{~K}$ the extrapolated values of grain-boundary and triple junction diffusivities are $2 \times 10^{-23}$ and $4 \times 10^{-20} \mathrm{~m}^{2} \mathrm{~s}^{-1}$ respectively [32]. Therefore, at this temperature, only triple junction diffusion is expected to be appreciable. Thus, asymmetry in the diffusivities of the

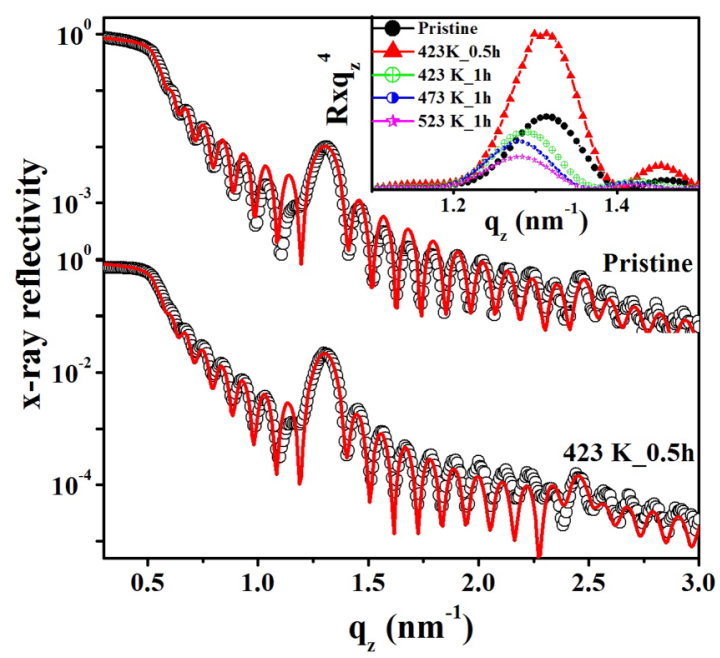

FIG. 5. X-ray reflectivity of $\mathrm{Cu} / \mathrm{Ni}$ multilayer annealed at $423 \mathrm{~K}$ for $0.5 \mathrm{~h}$. Inset compares the reflectivity at the first Bragg peak of samples annealed at different temperatures on a linear scale. two constituents cannot be the cause of the observed interface sharpening in the present study.

We propose a mechanism for the observed sharpening of the interfaces at $423 \mathrm{~K}$, in which fast diffusion along the shortcircuit paths like triple junctions plays a dominant role. It may be noted that in as-deposited multilayer a certain amount of intermixing exists at the interfaces of the two constituents as a consequence of the finite kinetic energy of the impinging atoms which make some diffusive jumps before settling down to a lattice site. The extent of intermixing will depend on the activation energy involved in a diffusive jump. The smaller the activation energy the larger the intermixing. Since the activation energy for diffusive jumps along a triple junction is orders of magnitude lower than that for grain-boundary or volume diffusion, the intermixing between $\mathrm{Cu}$ and $\mathrm{Ni}$ across a grain or a grain boundary should be much smaller than that across a triple junction line which intersects the interface between two $\mathrm{Ni}$ and $\mathrm{Cu}$ layers. The situation is depicted schematically in Fig. 6(a). The interface roughness has two contributions: the correlated part $\sigma_{c}$ which propagates from the substrate itself, and the uncorrelated part $\sigma_{u}$ [4]. The uncorrelated part in turn consists of a topological term due to the stochastic nature of the thin-film deposition $\left(\sigma_{u T}\right)$ and the interdiffusion term $\left(\sigma_{u D}\right)$. Thus the total roughness can be written as

$$
\sigma_{T}^{2}=\sigma_{c}^{2}+\sigma_{u}^{2},
$$

where

$$
\sigma_{u}^{2}=\sigma_{u T}^{2}+\sigma_{u D}^{2}
$$

Annealing at $423 \mathrm{~K}$ causes intermixing between $\mathrm{Cu}$ and Ni layers through fast interdiffusion along those triple junction lines which intersect the interfaces. Due to orders of magnitude higher triple junction diffusivity as compared to grain-boundary or volume diffusivities at this temperature, one expects type-C diffusion kinetics as per Harrison's classification [34]. Once composition along such triple junctions homogenizes, the contribution of triple junctions to $\sigma_{\mathrm{uD}}^{2}$ will disappear [Fig. 6(b)]. Instead, as a result a small decrease in the scattering contrast between $\mathrm{Cu}$ and $\mathrm{Ni}$ layers will appear. The net result of this is an effective sharpening of the interfaces. Continued annealing at $423 \mathrm{~K}$ will result in further composition variation along the triple junctions which do not intersect the interfaces. This should result in a decrease in the scattering contrast between adjacent layers, resulting in a decrease in the height of the Bragg peak. Annealing at still higher temperatures of 473 and $523 \mathrm{~K}$ at which grainboundary diffusion becomes appreciable, interface roughness will increase due to partial intermixing along grain boundaries. Indeed the diffusivities calculated from the decrease in the height of the first-order Bragg peak at 473 and $523 \mathrm{~K}$ lies close to the extrapolated value of grain-boundary diffusion as determined in the literature [32].

It may be noted that the diffusivities as obtained in the present case show a small decrease with increasing temperature. This happens because of the transient nature of diffusion in this temperature-time regime and the fact that a single sample is isochronally annealed at successively higher temperatures and the calculated diffusivities are the average 

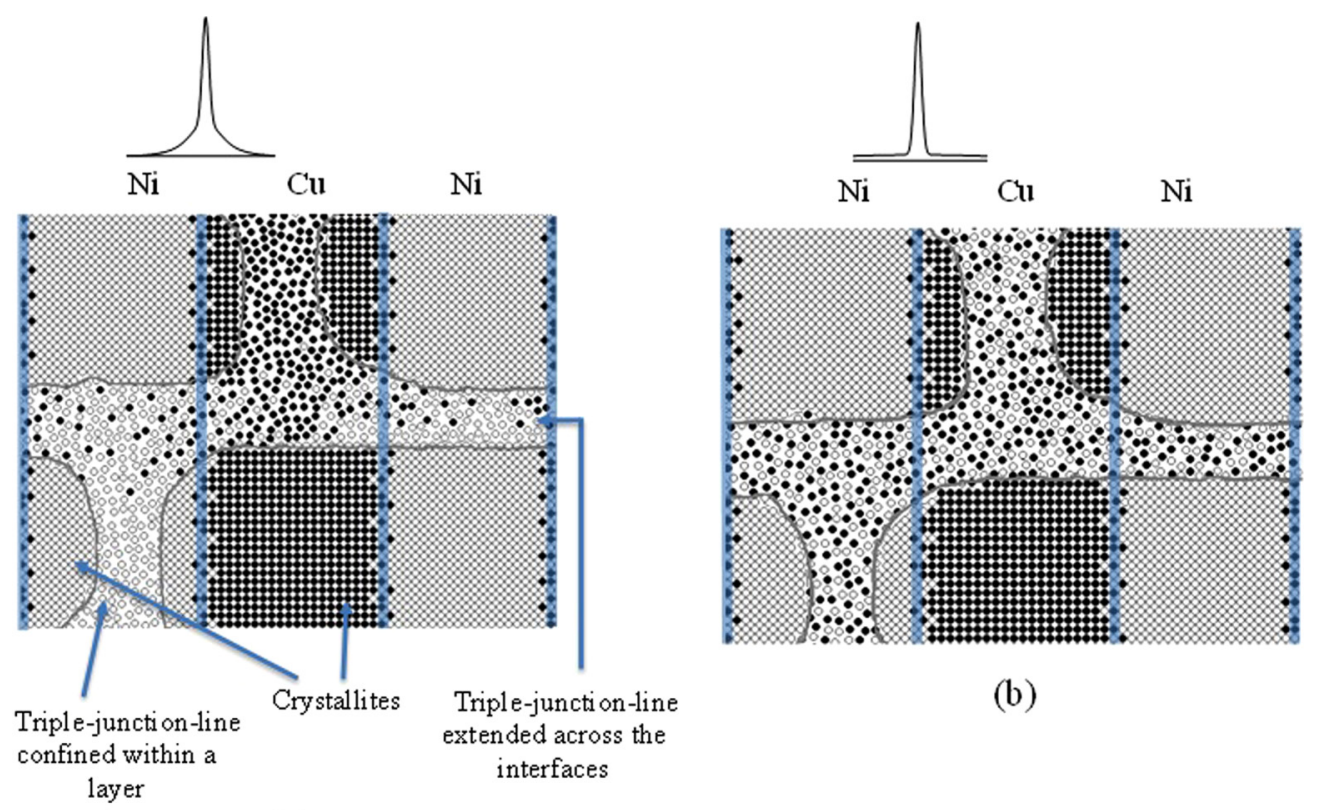

(b)

(a)

FIG. 6. Schematic of the structure of the multilayer (a) before and (b) after annealing at a temperature at which interface sharpening has been observed. At this temperature, interdiffusion across $\mathrm{Cu} / \mathrm{Ni}$ interfaces inside the crystallites is negligible, while in triple junction regions, a complete homogenization takes place, as described in the text. Open circles represent $\mathrm{Ni}$ atoms, while filled circles represent $\mathrm{Cu}$ atoms. Typical triple-junction line extending across several $\mathrm{Ni}$ and $\mathrm{Cu}$ layers, and those confined within a single $\mathrm{Ni}$ or $\mathrm{Cu}$ layer are indicated by arrows. Due to limitation with the projection on 2D plane, grain-boundary regions could not be depicted. It may be noted that there is a partial epitaxy between $\mathrm{Cu}$ and $\mathrm{Ni}$ layers with the average crystallite size of $9.7 \mathrm{~nm}$, thus a typical crystallite extends over several layers. Diffusion profile across the interfaces is also shown.

diffusivities over the annealing period. As discussed above, at $423 \mathrm{~K}$ observed diffusivity is intermediate between triple junction and grain-boundary diffusivities. At $473 \mathrm{~K}$ the diffusion along triple junctions is already saturated and further diffusion occurs along the grain boundaries. During annealing at $523 \mathrm{~K}$, initially diffusion occurs along grain boundaries and once the grain boundaries are saturated, further diffusion occurs through grains. Therefore the average diffusivity at this temperature is intermediate between that for grain-boundary and volume diffusivities.

\section{B. ${ }^{57} \mathrm{Fe} /{ }^{\text {natural }} \mathrm{Fe}$ multilayer}

In order to conclusively exclude the possibility of some concentration dependent diffusivity being the cause of interface sharpening in the $\mathrm{Cu} / \mathrm{Ni}$ multilayer, the effect of thermal annealing on the isotopic interfaces in a multilayer consisting of nanocrystalline ${ }^{57} \mathrm{Fe} /{ }^{\text {natural }} \mathrm{Fe}$ was studied using nuclear resonance reflectivity (NRR). Since chemically the isotopic multilayer is a single layer of $\mathrm{Fe}$, there is no question of any concentration dependent diffusivity in the system. When the energy of the $\mathrm{x}$ rays is tuned to the Mössbauer transition of ${ }^{57} \mathrm{Fe}$ nuclei, nuclear resonance scattering from ${ }^{57} \mathrm{Fe}$ causes a strong scattering contrast between ${ }^{57} \mathrm{Fe}$ and ${ }^{\text {natural }} \mathrm{Fe}$. This results in Bragg peaks in the nuclear resonance reflectivity corresponding to the isotopic periodicity in the multilayer [30]. The height of a Bragg peak is proportional to the roughness of the isotopic interfaces and has been used in the literature to study self-diffusion of $\mathrm{Fe}[30,35,36]$. Figure 7(a) gives the nuclear resonance reflectivity of an isotopic multi- layer having structure $\left[{ }^{57} \mathrm{Fe}(3.2 \mathrm{~nm}) /{ }^{\mathrm{natural}} \mathrm{Fe}(3.2 \mathrm{~nm})\right]_{10}$ as a function of annealing at different temperatures for $1 \mathrm{~h}$ each. The reflectivity patterns have been fitted using the software REFTIM [37] and the simulated results are shown as continuous curves. One expects an error function concentration profile at the interface, however, for simplicity a linear concentration profile was assumed. The calculated concentration profile of ${ }^{57} \mathrm{Fe}$ is shown in Fig. 7(b). One can see that annealing at $523 \mathrm{~K}$ results in an increase in the reflectivity at the first Bragg peak, and a sharpening of the isotopic interface as seen from Fig. 7(b). In the isotopic multilayer, since both the constituents are chemically identical, the mechanism of concentration dependent diffusivities proposed in the earlier studies can be ruled out. In this system, the mechanism proposed in the earlier section is the only possibility. Negative diffusion length at $523 \mathrm{~K}$ as calculated from the height of the Bragg peak comes out to be $-0.61 \mathrm{~nm}$. The calculated value of diffusivity at 573 and $623 \mathrm{~K}$ comes out to be $8.9 \times 10^{-23}$ and $1.37 \times 10^{-22} \mathrm{~m}^{2} \mathrm{~s}^{-1}$ respectively. The present values lie in between those for volume [38] and grain-boundary diffusivities $[39,40]$ and are close to the values for self-diffusion of $\mathrm{Fe}$ in nanocrystalline film as obtained in some earlier study [41].

It may be noted that grain-boundary diffusivities reported in the literature are for polycrystalline $\mathrm{Fe}$. The same in nanocrystalline film with a higher density of grain boundaries is expected to be higher. Thus, it may be noted that the grain-boundary diffusion even at $523 \mathrm{~K}$ is not negligible and should cause an increase in the interface roughness. However, the effective sharpening of the interfaces associated 
(a)

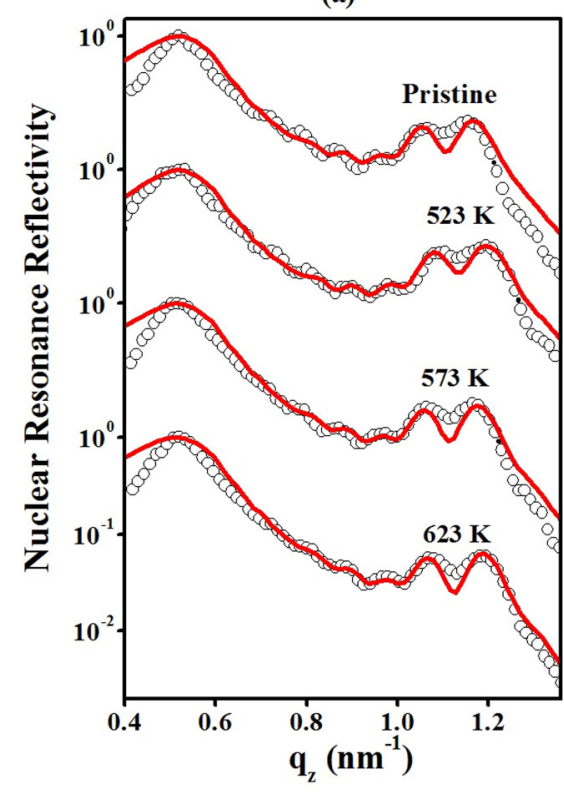

(b)

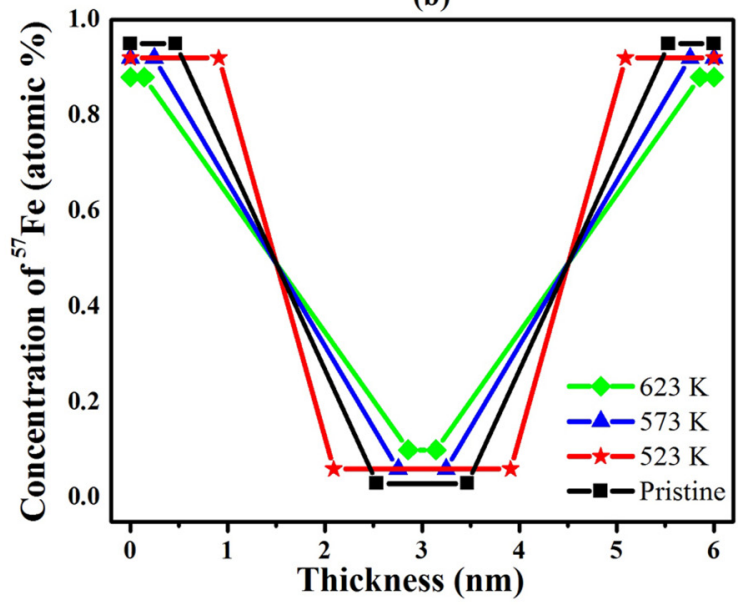

FIG. 7. (a) Nuclear resonance reflectivity of isotopic multilayer substrate $/\left[{ }^{57} \mathrm{Fe}(3.2 \mathrm{~nm}) /{ }^{\mathrm{natural}} \mathrm{Fe}(3.2 \mathrm{~nm})\right]_{10}$ after annealing at different temperatures for $1 \mathrm{~h}$ each. The continuous curve represents the best fit to the experimental data, assuming a linear concentration profile across the isotopic interface. (b) The corresponding concentration profiles as obtained from the best fit of the data.

with homogenization of isotopic composition along the triple junctions dominates, causing an overall decrease in the interface roughness. In order to further ascertain this point, another isotopic multilayer having structure substrate $/{ }^{\text {natural }} \mathrm{Fe}(50 \mathrm{~nm}) /\left[{ }^{57} \mathrm{Fe}(1.8 \mathrm{~nm}) /{ }^{\text {natural }} \mathrm{Fe}(3.8 \mathrm{~nm})\right]_{10}$ was prepared by keeping the substrate temperature at $573 \mathrm{~K}$. Nuclear resonance reflectivity of the as-prepared as well as $573 \mathrm{~K}$ annealed multilayer is shown in Fig. 8. The negative diffusion length as calculated from the height of the Bragg peak comes out to be $-0.55 \mathrm{~nm}$, which is less than the value obtained after annealing of room-temperature multilayer at $523 \mathrm{~K}$. The result can be understood by considering that grain-boundary diffusion at $573 \mathrm{~K}$ is higher than that at 523

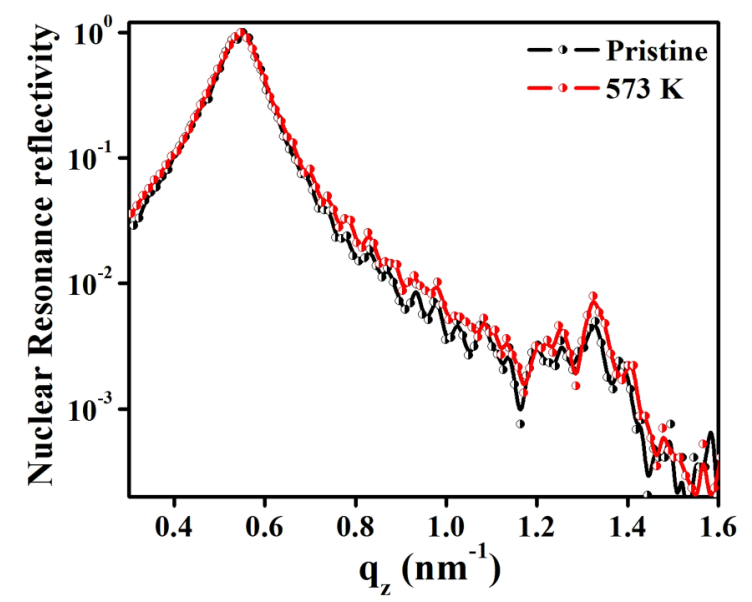

FIG. 8. Nuclear resonance reflectivity of isotopic multilayer substrate $/{ }^{\text {natural }} \mathrm{Fe}(50 \mathrm{~nm}) /\left[{ }^{57} \mathrm{Fe}(1.8 \mathrm{~nm}) /{ }^{\text {natural }} \mathrm{Fe}(3.8 \mathrm{~nm})\right]_{10}$ prepared at $573 \mathrm{~K}$ in pristine form and after annealing at $573 \mathrm{~K}$ for $1 \mathrm{~h}$.
$\mathrm{K}$, and therefore the roughening effect of grain-boundary diffusion is higher in this case.

\section{CONCLUSIONS}

In conclusion, the evolution of interfaces in $\mathrm{Cu} / \mathrm{Ni}$ multilayer with thermal annealing has been studied using anomalous $\mathrm{x}$-ray reflectivity. It is found that at the relatively low temperature of $423 \mathrm{~K}$, at which both volume as well as grainboundary diffusion are low, thermal annealing results in the sharpening of the interfaces, causing interface roughnesses to decrease by about 38\%. Annealing at higher temperatures in the range $473-523 \mathrm{~K}$ results in interdiffusion and the calculated diffusivities agree with extrapolated values of grain-boundary diffusivities from the literature. It is suggested that fast diffusion occurring along the short-circuit paths like triple junctions causes an effective decrease in the interface roughnesses. Sharpening of the interfaces is expected to occur in the temperature regime in which diffusion along shortcircuit paths is fast enough, while the normal diffusion is significantly lower. The observed effect is general in nature and different from that observed in systems with a large asymmetry in the diffusivities of the two constituents $[15,16]$. The general nature of the effect is conclusively demonstrated by studying the evolution of isotopic interfaces in ${ }^{57} \mathrm{Fe} /{ }^{\text {natural }} \mathrm{Fe}$ multilayers using nuclear resonance reflectivity. Sharpening of isotopic interfaces is observed upon annealing at $523 \mathrm{~K}$. In the isotopic multilayer, since both the constituents are chemically identical, the mechanism of concentration dependent diffusivities proposed in the earlier studies can be ruled out.

\section{ACKNOWLEDGMENTS}

A.T. is a senior research fellow of CSIR, India. This work was partially supported by Science and Engineering Research 
Board through Project No. SB/S2/CMP-007/2013. Support for the experiment at PETRA-III, Germany, was provided by the Department of Science and Technology, Government of India through Jawaharlal Nehru Centre for Advanced Scientific Research. Fitting of NRR data was done using the program REFTIM, kindly provided by Professor M. A. Andreeva. Thanks are due to J. Dwivedi and L. Behra for their help in deposition of multilayer structures and for XRD measurements. The help of A. Khooha during measurements at BL-16 of Indus-2 is thankfully acknowledged.
[1] R. Galceran, L. Balcells, C. Martinez-Boubeta, B. Bozzo, J. Cisneros-Fernández, M. de la Mata, C. Magén, J. Arbiol, J. Tornos, F. A. Cuellar, Z. Sefrioui, A. Cebollada, F. Golmar, L. E. Hueso, F. Casanova, J. Santamaría, and B. Martinez, Phys. Rev. B 92, 094428 (2015).

[2] K. O'Grady, L. E. Fernandez-Outon, and G. Vallejo-Fernandez, J. Magn. Magn. 322, 883 (2010).

[3] H. Yang, A. Thiaville, S. Rohart, A. Fert, and M. Chshiev, Phys. Rev. Lett. 115, 267210 (2015).

[4] D. E. Savage, J. Kleiner, N. Schimke, Y. H. Phang, T. Jankowski, J. Jacobs, R. Kariotis, and M. G. Lagally, J. Appl. Phys. 69, 1411 (1991).

[5] R. Maruyama, D. Yamazaki, T. Ebisawa, and K. Soyama, J. Appl. Phys. 105, 083527 (2010).

[6] A. T. G. Pym, A. Lamperti, B. K. Tanner, T. Dimopoulos, M. Rührig, and J. Wecker, Appl. Phys. Lett. 88, 162505 (2006).

[7] S. Ikeda, K. Miura, H. Yamamoto, K. Mizunuma, H. D. Gan, M. Endo, S. Kanai, J. Hayakawa, F. Matsukura, and H. Ohno, Nat. Mater. 9, 721 (2010).

[8] C. Rizal and E. E. Fullerton, J. Phys. D: Appl. Phys. 50, 355002 (2017).

[9] F. Richomme, J. Teillet, A. Fnidiki, and W. Keune, Phys. Rev. B 64, 094415 (2001).

[10] T. Das, P. D. Kulkarni, S. C. Purandare, H. C. Barshilia, S. Bhattacharyya, and P. Chowdhury, Sci. Rep. 4, 5328 (2014).

[11] Z. Erdélyi and D. L. Beke, J. Mater. Sci. 46, 6465 (2011).

[12] R. Würschum, P. Farber, R. Dittmar, P. Scharwaechter, W. Frank, and H. E. Schaefer, Phys. Rev. Lett. 79, 4918 (1997).

[13] A. Tayal, M. Gupta, A. Gupta, P. Rajput, and J. Stahn, Phys. Rev. B 92, 054109 (2015).

[14] A. Gupta, D. Kumar, and V. Phatak, Phys. Rev. B 81, 155402 (2010).

[15] Z. Erdélyi, M. Sladecek, L. M. Stadler, I. Zizak, G. A. Langer, M. Kis-Varga, D. L. Beke, and B. Sepiol, Science 306, 1913 (2004).

[16] Z. Balogh, M. R. Chellali, G. H. Greiwe, G. Schmitz, and Z. Erdélyi, Appl. Phys. Lett. 99, 181902 (2011).

[17] J.-M. Roussel and P. Bellon, Phys. Rev. B 73, 085403 (2006).

[18] M. C. Benoudia, F. Gao, J.-M. Roussel, S. Labat, M. Gailhanou, O. Thomas, D. L. Beke, Z. Erdélyi, G. Langer, A. Csik, and M. Kis-Varga, Phys. Rev. B 85, 235404 (2012).
[19] Z. Balogh, M. R. Chellali, P. Stender, and G. Schmitz, Defect Diffus. Forum 353, 177 (2014).

[20] Z. Erdélyi, I. A. Szabó, and D. L. Beke, Phys. Rev. Lett. 89, 165901 (2002).

[21] L. Priester, Grain Boundaries: From Theory to Engineering (Springer, Dordrecht, 2013), p. 172.

[22] J. Askill, Tracer Diffusion Data for Metals, Alloys and Simple Oxides (Springer, New York, 1970).

[23] R. F. Baluffi, Met. Trans. B 13, 527 (1982).

[24] N. L. Peterson, J. Vac. Sci. Technol. A 4, 3066 (1986).

[25] T. Frolov and Y. Mishin, Phys. Rev. B 79, 174110 (2009).

[26] Y. Chen and C. A. Schuh, Scr. Mater. 57, 253 (2007).

[27] B. L. Henke, E. M. Gullikson, and J. C. Davis, At. Data Nucl. Data Tables 54, 181 (1993).

[28] L. G. Parratt, Phys. Rev. 95, 359 (1954).

[29] G. Palumbo, S. J. Thorpe, and K. T. Aust, Scr. Metall. Mater. 24, 1347 (1990).

[30] A. Gupta, M. Gupta, S. Chakravarty, R. Rüffer, H.-C. Wille, and O. Leupold, Phys. Rev. B 72, 014207 (2005).

[31] T. Mizoguchi, S. Tanabe, and M. Murata, J. Magn. Magn. 126, 96 (1993).

[32] M. R. Chellali, Z. Balogh, H. Bouchikhaoui, R. Schlesiger, P. Stender, L. Zheng, and G. Schmitz, Nano Lett. 12, 3448 (2012).

[33] A. Almazouzi, M.-P. Macht, V. Naundorf, and G. Neumann, Phys. Rev. B 54, 857 (1996).

[34] C. Herzig and Y. Mishin, in Diffusion in Condensed Matter: Methods, Materials, Models, edited by P. Heitjans and J. Kärger (Springer, Berlin, 2005), p. 337.

[35] R. Gupta, A. Gupta, W. Leitenberger, and R. Rüffer, Phys. Rev. B 85, 075401 (2012).

[36] M. Rennhofer, B. Sepiol, M. Sladecek, D. Kmiec, S. Stankov, G. Vogl, M. Kozlowski, R. Kozubski, A. Vantomme, J. Meersschaut, R. Rüffer, and A. Gupta, Phys. Rev. B 74, 104301 (2006).

[37] M. A. Andreeva, Hyperfine Interact. 185, 17 (2008).

[38] M. Lübbehusen and H. Mehrer, Acta Metall. Mater. 38, 283 (1990).

[39] Y. Iijima, J. Phase Equilib. Diffus. 26, 466 (2005).

[40] H. Tanimoto, P. Farber, R. Würschum, R. Z. Valiev, and H.-E. Schäfer, Nanostruct. Mater. 12, 681 (1999).

[41] S. Chakravarty, N. Shukla, A. Devishvili, A. Vorobiev and G. Amarendra, Mater. Res. Express 3, 085001 (2016). 